\title{
Electrical Characterization and Modeling of 1T-1R RRAM arrays with Amorphous and Poly-crystalline $\mathrm{HfO}_{2}$
}

\author{
Alessandro Grossi ${ }^{1, *}$, Cristian Zambelli ${ }^{1}$, Piero Olivo ${ }^{1}$, Alberto Crespo-Yepes, ${ }^{2}$, Rosana Rodríguez ${ }^{2}$, Eduardo Perez ${ }^{3}$, Christian \\ Wenger ${ }^{3}$ \\ Università degli Studi di Ferrara, Dip. di Ingegneria, Via Saragat 1, Ferrara, 44122, Italy
}

\begin{abstract}
In this work, a comparison between 1T-1R RRAM arrays, manufactured either with amorphous or poly-crystalline Metal-InsulatorMetal cells, is reported in terms of performance, reliability, Set/Reset operations energy requirements, intra-cell and inter-cell variability during 10k endurance cycles and 100k read disturb cycles. The modeling of the 1T-1R RRAM array cells has been performed with two different approaches: i) a physical model like the Quantum Point Contact (QPC) model was used to find the relationship between the reliability properties observed during the endurance and the read disturb tests with the conductive filament properties; ii) a compact model to be exploited in circuit simulations tools which models the I-V characteristics of each memory cells technology.
\end{abstract}

Keywords: RRAM array, forming, read window, energy saving

\section{Introduction}

Resistive Random Access Memories (RRAM) technology gathered significant interest for several applications [1, 2, 3]. RRAM behavior is based on the possibility of electrically modifying the conductance of a Metal-Insulator-Metal (MIM) stack: the Set operation moves the cell in a low resistive state (LRS), whereas Reset brings the cell in a high resistive state (HRS) $[4,5]$. To activate such a switching behavior, some technologies require a preliminary Forming operation [6, 7, 8].

The choice of a proper Metal-Insulator-Metal (MIM) technology for RRAM cells, exhibiting good uniformity and low switching voltages, is still a key issue for array structures fabrication and reliable electrical operation [9]. Such a process step is mandatory to bring this technology to a maturity level. In this work, a comparison between 1T-1R RRAM 4Kbits arrays manufactured either with amorphous [5] or poly-crystalline [10] $\mathrm{HfO}_{2}$ is performed. In amorphous $\mathrm{HfO}_{2}$ the conduction mainly occurs through a conductive filament with a variable concentration of defects, whereas in poly-crystalline $\mathrm{HfO}_{2}$ the conduction occurs only through grain boundaries with a very low defect concentration. The differences in terms of conduction properties and defect concentrations translate into different switching properties [9], with several implications on inter-cell variability (variations between cells) and intra-cell variability (cycleto-cycle variations of any given cell).

\footnotetext{
${ }^{*}$ Corresponding author

Email address: alessandro.grossi@unife.it (Alessandro Grossi)

${ }^{1}$ Università degli Studi di Ferrara, Dip. di Ingegneria, Via Saragat 1, 44122 Ferrara, Italy

${ }^{2}$ Universitat Autònoma de Barcelona (UAB), Departament d'Enginyeria Electrònica, 08193 Bellaterra, Spain

${ }^{3}$ IHP, Im Technologiepark 25, 15236 Frankfurt (Oder), Germany
}

In this work, that is an extended yet complete picture of the results presented in [11], a comparison in terms of performance, reliability, Set/Reset operations energy requirements, intra-cell and inter-cell variability during $10 \mathrm{k}$ endurance cycles is reported. In addition to the previously presented results, $100 \mathrm{k}$ read disturb cycles were performed to deepen the understanding of the reliability of each technology. Moreover, to understand the relationship between the reliability properties observed during the endurance and read disturb tests and the conductive filament properties, Quantum Point Contact (QPC) modeling [12] was used, since it allows to correctly represent the measured $\mathrm{I}-\mathrm{V}$ characteristics independently from the conduction mechanism. Even if the QPC allows to model the conductive filaments properties taking into account the cell-to-cell variability, it offers a technology description that sometimes is complex to be implemented in circuit simulation tools. To this extent, an equivalent circuit model able to offer a simpler description of the devices was applied and validated on both MIM technologies. The memory cells used in this work can be modelled using a diode-resistance equivalent circuit model. The model parameters extracted from the fittings of experimental I-V curves can provide additional information about electrical properties of the memory cells to be exploited in the design of RRAM arrays.

\section{Experimental Setup}

The 1T-1R memory cells in the 4kbits arrays are constituted by a select NMOS transistor manufactured with a $0.25 \mu \mathrm{m} \mathrm{BiC}$ MOS technology whose drain is in series to a MIM stack. The wordline (WL) voltage applied to the gate of the NMOS transistor allows setting the cell current compliance. The crosssectional Scanning Transmission Electron Microscopy (STEM) 


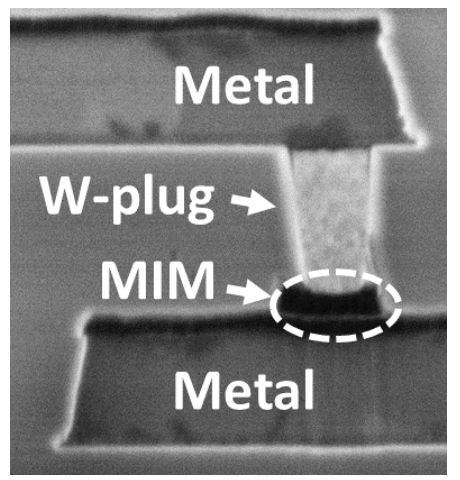

(a)

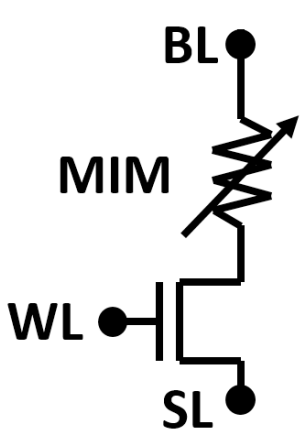

(b)

Figure 1: Cross-sectional STEM image (a) and schematic (b) of the 1T-1R cell integrated in the arrays.

Table 1: Forming, Set, Reset and Read Voltage Parameters.

\begin{tabular}{|l|c|c|c|}
\hline Operation & $\mathrm{V}_{S L}[\mathrm{~V}]$ & $\mathrm{V}_{B L}[\mathrm{~V}]$ & $\mathrm{V}_{W L}[\mathrm{~V}]$ \\
\hline Forming & 0 & $2-3.2$ & 1.5 \\
Set & 0 & $0.2-3.2$ & 1.5 \\
Reset & $0.2-3.2$ & 0 & $2.5(\mathrm{~A}) / 2.8(\mathrm{P})$ \\
Read & 0 & 0.2 & 1.5 \\
\hline
\end{tabular}

image of the cell and the 1T-1R cell schematic are reported in Fig. 1. The variable MIM resistor is composed by $150 \mathrm{~nm}$ TiN top and bottom electrode layers deposited by magnetron sputtering, a $7 \mathrm{~nm}$ Ti layer, and a $8 \mathrm{~nm} \mathrm{HfO}_{2}$ layer deposited with two different Atomic Vapour Deposition (AVD) processes resulting either in amorphous (A) or poly-crystalline (P) $\mathrm{HfO}_{2}$ films, respectively. The resistor area is equal to $0.4 \mu \mathrm{m}^{2}$. For amorphous films it has been integrated also a resistor with larger area that shows improved reliability and performance (i.e., 1 $\mu \mathrm{m}^{2}$ ) [4]. The Forming/Set/Reset operations on the arrays were performed by using an Incremental Pulse and Verify algorithm. The bitline (BL), sourceline (SL) and WL voltages applied during Forming, Set, Reset and Read operations are reported in Tab. 1. Reset operations were performed by applying the highest WL voltage available $(2.8 \mathrm{~V}$ on array $\mathrm{A}$ and $2.5 \mathrm{~V}$ on array $\mathrm{P}$ ) to maximize the cells switching yield while avoiding the breakdown of the MIM [13]. Pulses were applied during Forming by increasing $\mathrm{V}_{B L}$ with $\Delta \mathrm{V}_{B L}=0.01 \mathrm{~V}$, whereas during Set and Reset $\Delta \mathrm{V}_{B L}=0.1 \mathrm{~V}$ and $\Delta \mathrm{V}_{S L}=0.1 \mathrm{~V}$ have been used, respectively. Each pulse featured a duration of $10 \mu \mathrm{s}$, with a rise/fall time of $1 \mu$ s to avoid overshoot issues. Set operation was stopped on a cell when the read-verify current reached $20 \mu \mathrm{A}$, whereas Reset was stopped when $10 \mu \mathrm{A}$ was reached. Forming, Set and Reset BL/SL voltages necessary to reach the requested read-verify current targets are extracted from the characterization data and labelled as $\mathrm{V}_{F O R M}, \mathrm{~V}_{S E T}$ and $\mathrm{V}_{R E S}$, respectively.

\section{Experimental Results}

Arrays using A- $\mathrm{HfO}_{2}$ (A-array) with resistor area of $0.4 \mu \mathrm{m}^{2}$, $1 \mu \mathrm{m}^{2}$ and P-HfO ${ }_{2}$ (P-array) resulted in a Forming Yield (calculated as the cell percentage showing a read verify current

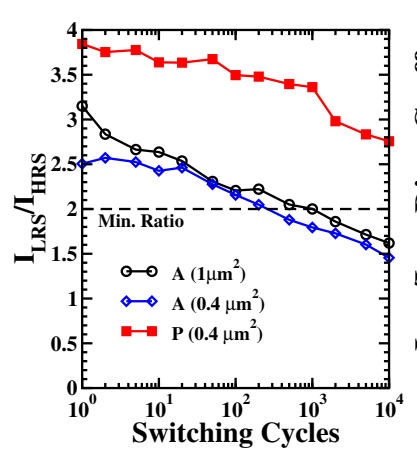

(a)

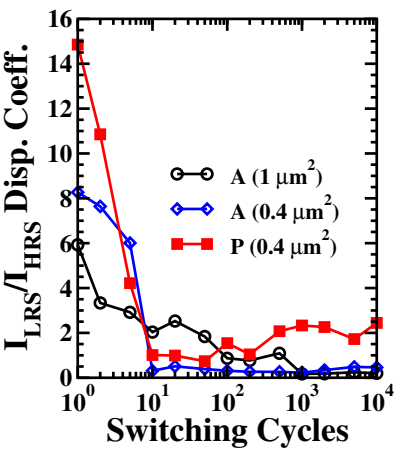

(b)
Figure 2: $\mathrm{I}_{L R S} / \mathrm{I}_{H R S}$ current ratio average values (a) and dispersion coefficients (b) calculated during cycling.

after forming $\mathrm{I}_{\text {read }} \geq 20 \mu \mathrm{A}$ ) of $58 \%, 90 \%$ and $95 \%$, respectively. Fig. 2 shows the average current ratios between Low Resistive State (LRS) and High Resistive State (HRS) read currents $\left(\mathrm{I}_{L R S} / \mathrm{I}_{H R S}\right)$, calculated on the entire cells population during SET/RESET cycling at $\mathrm{V}_{\text {read }}=0.2 \mathrm{~V}$ on A-array and Parray, and their relative dispersion coefficient. The minimum current ratio that allows to correcly discriminate between HRS and LRS, defined as $\mathrm{I}_{L R S} / \mathrm{I}_{H R S}>2$, is indicated for comparison [5]. The average ratios of A-arrays with resistor area of $0.4 \mu \mathrm{m}^{2}$ and $1 \mu \mathrm{m}^{2}$ go under the minimum ratio limit after 200 and $1 \mathrm{k}$ cycles, respectively. To evaluate the cell-to-cell variability the dispersion coefficient of $I_{L R S}$ and $I_{H R S}$ distributions, defined as $\left(\sigma^{2} / \mu\right)$, has been used. P-array showed higher Ratio $(\approx 2.8)$ even after $10 \mathrm{k}$ cycles, but also a higher dispersion coefficient after Forming (i.e., cycle 1). The grain boundaries conduction mechanism in the poly-crystalline $\mathrm{HfO}_{2}$ structure could be the reason of the higher cell-to-cell variability in P-arrays [14]. Aarray with resistor area of $1 \mu \mathrm{m}^{2}$ shows a slightly higher average ratio than A-array with resistor area of $0.4 \mu \mathrm{m}^{2}$.

Fig. 3 shows a comparison between $\mathrm{I}_{L R S}$ and $\mathrm{I}_{H R S}$ cumulative distributions measured at cycle 1 and after the endurance test: A-arrays show more compact distributions at cycle 1, however after the endurance test P-array shows a higher percentage of correctly switching cells reaching the Set/Reset verify targets. $\mathrm{I}_{H R S}$ cumulative distribution in P-array show a longer tail at cycle 1 compared to A-arrays. After 10k cycles only an increase of the tail in P-array can be observed whereas on A-arrays a strong shift of the distributions towards higher currents occurs, resulting in a higher number of cells not reaching the Reset threshold. $\mathrm{I}_{H R S}$ cumulative distribution in A-array with with resistor area of $1 \mu \mathrm{m}^{2}$ shows lower currents at cycle 1 than Aarrays with resistor area of $0.4 \mu \mathrm{m}^{2}$, however after 10k cycles $\mathrm{I}_{H R S}$ cumulative distributions are very similar. $\mathrm{In} \mathrm{I}_{L R S}$ cumulative distributions a tail creation of cells not able to reach the set threshold can be observed on P-arrays after 10k cycles, whereas on A-arrays a strong shift of the distributions towards lower currents occurs, resulting in a higher number of cells not reaching the Set threshold especially when cells with resistor area of $0.4 \mu \mathrm{m}^{2}$ are considered. A-array with resistor area of $0.4 \mu \mathrm{m}^{2}$ shows a high number of cells not reaching the Set threshold 


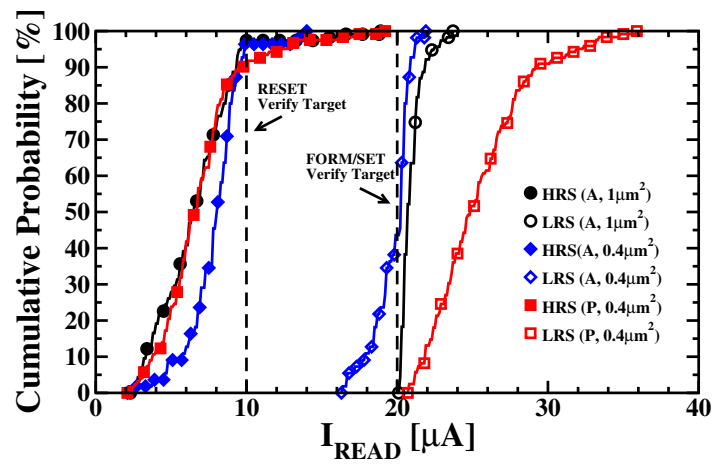

(a)

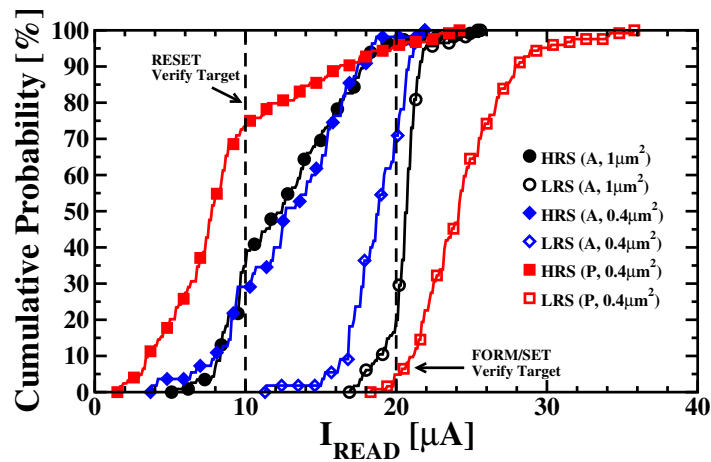

(b)

Figure 3: $\mathrm{I}_{H R S}$ and $\mathrm{I}_{L R S}$ cumulative distributions at cycle 1 (a) and at cycle 10k (b).

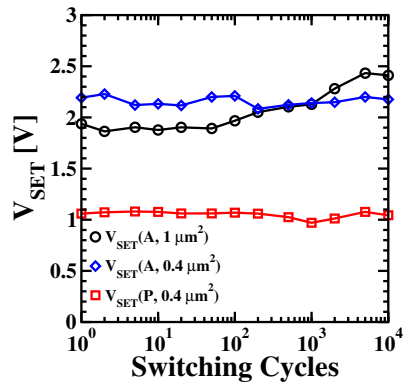

(a)

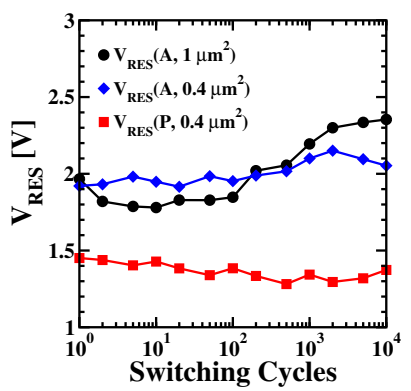

(c)

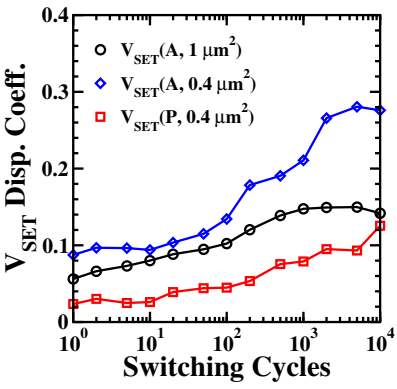

(b)

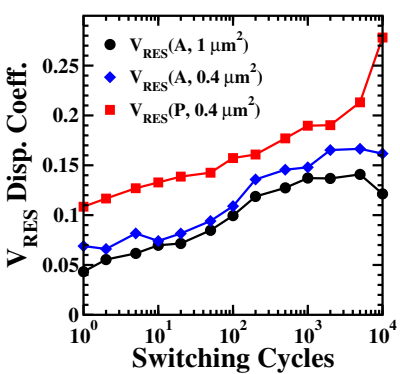

(d)
Figure 4: $\mathrm{V}_{S E T}$ and $\mathrm{V}_{R E S}$ average values $(\mathrm{a}, \mathrm{b})$ and dispersion coefficients (c,d) calculated during cycling.

even at cycle 1 .

Fig. 4 shows the average Set and Reset switching voltages $\left(\mathrm{V}_{S E T}, \mathrm{~V}_{R E S}\right)$ and their relative dispersion coefficients: lower
$\mathrm{V}_{S E T}$ and $\mathrm{V}_{\text {RES }}$ are required on $\mathrm{P}$-array which shows no variations during the endurance test, whereas $\mathrm{V}_{S E T}, \mathrm{~V}_{R E S}$ increase on A-arrays during cycling. $\mathrm{V}_{R E S}$ on $\mathrm{P}$-array shows the highest variability. A-arrays show similar behavior of the average $\mathrm{V}_{S E T}$ and $\mathrm{V}_{R E S}$ (a lower average $\mathrm{V}_{S E T}$ is observed on A-array with larger resistor area only up to 500 cycles), while a higher $\mathrm{V}_{S E T}$ and $\mathrm{V}_{R E S}$ dispersion can be observed in A-array with smaller resistor area.

Fig. 5 shows the cumulative distributions of Forming, Set and Reset switching voltages at cycle 1 and after the endurance test: Forming,Set and Reset algorithms starting point and last attempt are indicated, corresponding to the first and the last voltage pulse available in the incremental pulse and verify procedure. $\mathrm{P}$-array requires lower $\mathrm{V}_{S E T}$ and $\mathrm{V}_{R E S}$ but higher $\mathrm{V}_{F O R M}$ if compared to A-array with the same resistor area. A-array with larger resistor area requires higher $\mathrm{V}_{\text {FORM }}$, moreover it can be observed that $\approx 40 \%$ of the devices with smaller resistor area reached the forming threshold at $\mathrm{V}_{\text {FORM }}=2 \mathrm{~V}$, corresponding to the first attempt of the Forming Algorithm. Since P-array shows a more compact distribution on $\mathrm{V}_{S E T}$ and a larger $\mathrm{V}_{R E S}$ than $\mathrm{A}$ arrays, faster Set operation could be reliably used on P-array, whereas on Reset an incremental pulse with verify technique is required to ensure good reliability. A-arrays show large distributions on both $\mathrm{V}_{S E T}$ and $\mathrm{V}_{R E S}$, hence the adaptation of incremental pulse with verify techniques is mandatory on such arrays.

Fig. 6 shows the average energy required to perform Set and Reset operations on a single cell: P-array shows lower power consumption with a lower increase during cycling. A-arrays with different resistor area show similar power consumption during Reset operation, whereas a lower consumption during Set is observed on A-array with larger resistor area only up to 500 cycles. The overall energy required to create/disrupt the conductive filament during Set/Reset operations has been calculated as:

$$
E=\sum_{i=1}^{n} V_{\text {pulse }, i} * I_{\text {pulse }, i} * T_{\text {pulse }}+V_{\text {read }} * I_{\text {read }, i} * T_{\text {read }}
$$

Where $n$ is the number of reset pulses applied during incremental pulse operation, $V_{\text {pulse }, i}$ is the pulse voltage applied at step $i, I_{\text {pulse }, i}$ is the current flowing through RRAM cell during pulse $i$ application, $T_{\text {pulse }}=10 \mu \mathrm{s}$ is the pulse length, $V_{\text {read }}=0.2$ $\mathrm{V}$ is the read voltage applied during verify operation, $I_{\text {read }, i}$ is the current read during read verify step $i$, and $T_{\text {read }}=10 \mu \mathrm{s}$ is the verify pulse length.

In the considered RRAM cells the read signals has the same polarization of the Set operation (both pulses are applied on the $\mathrm{BL}$ ), hence the read disturb could only be a problem on cells in HRS state since a very long sequence of read pulses could slowly re-create the conductive filament, resulting into an undesired switch from HRS to LRS [13]. Read disturb has been evaluated only on cells in HRS state for each considered technology: Fig. 7 shows the average HRS read current and its relative standard deviation measured during 100k read operations. P-array shows the highest read current variation, confirming that on such technology due to the high leakage currents it is easier to create conductive paths. 


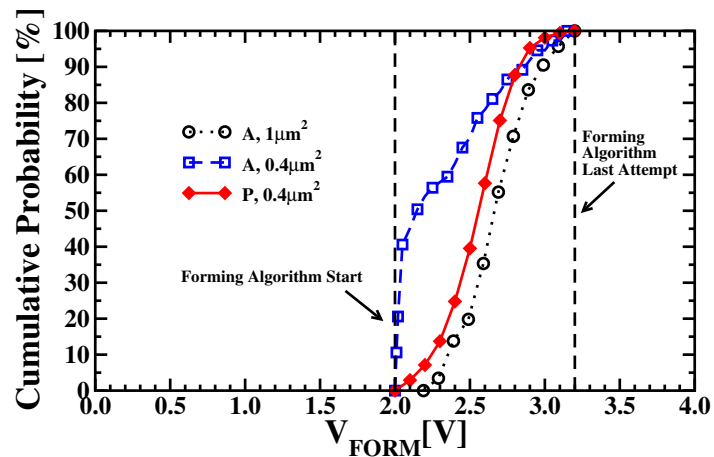

(a)

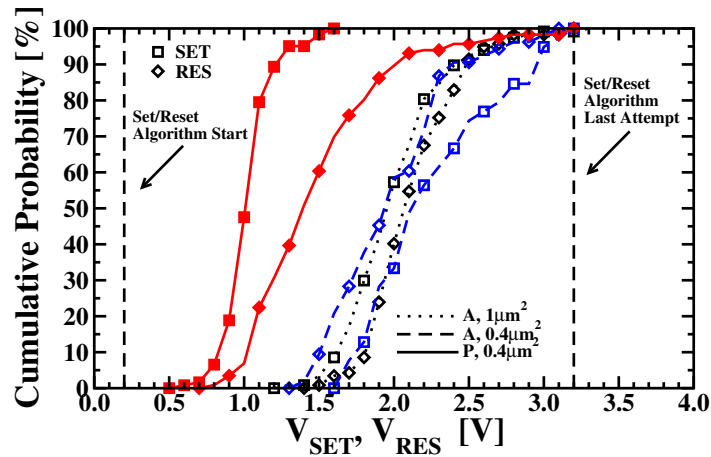

(b)

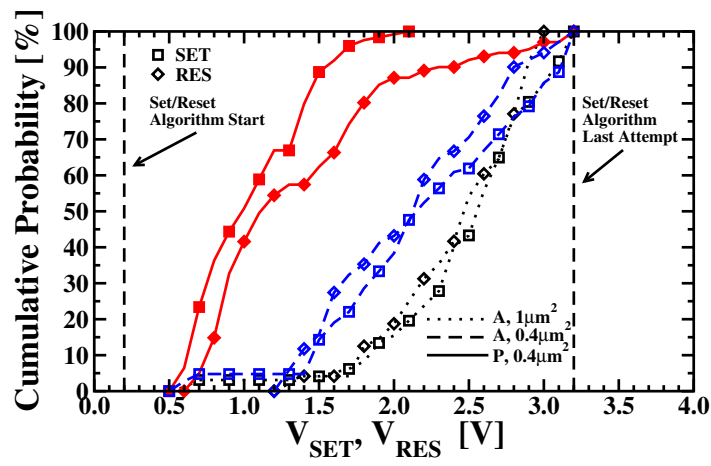

(c)

Figure 5: $\mathrm{V}_{F O R M}, \mathrm{~V}_{S E T}$ and $\mathrm{V}_{\text {RES }}$ cumulative distributions at cycle 1 (a) and at cycle $10 \mathrm{k}(\mathrm{b})$.

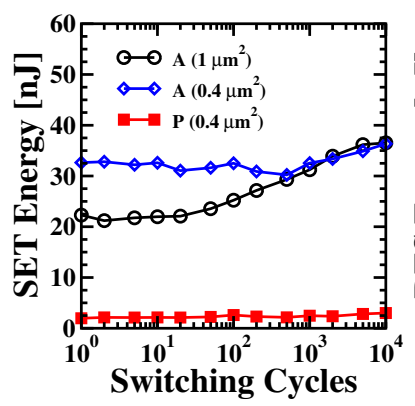

(a)

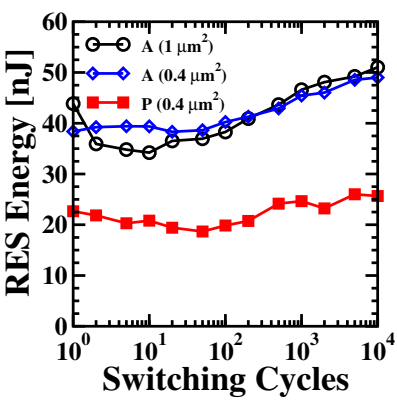

(b)
Figure 6: Energy required to perform Set (a) and Reset (b) operations as a function of the Set/Reset cycle number.

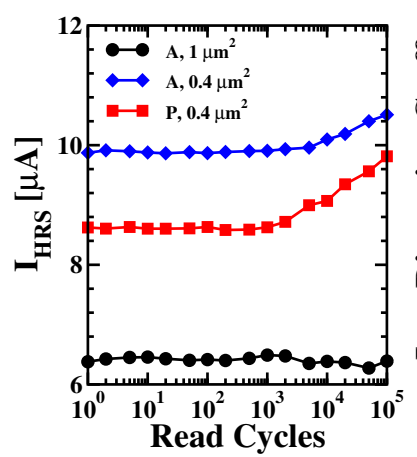

(a)

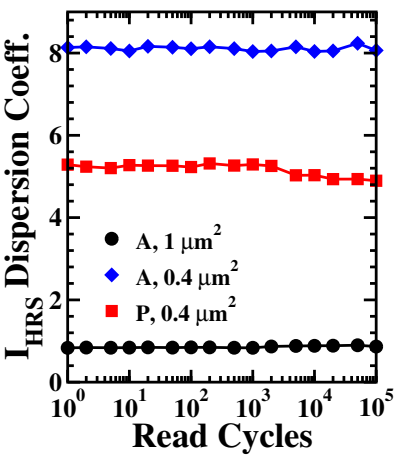

(b)
Figure 7: Average read current variation (a) and dispersion coefficient evolution (b) of HRS calculated during $100 \mathrm{k}$ read disturb pulses, with $V_{\text {pulse }}=0.2 \mathrm{~V}$.

\section{1T-1R cells modeling}

IV characteristics have been measured after-forming and modeled with two different approaches: in order to understand the differences on the conductive filament properties and variability QPC modeling has been used as in [15], while an equivalent circuit model [16] was used to obtain a description implementable in circuit simulation tools.

\section{1. $Q P C$ modeling}

Reset I-V characteristics measured after-forming were used to analyze the conductive filament properties through QPC model. HRS current is calculated according to the expression:

$$
I=\frac{2 e}{h} G / G_{0}\left(e V+\frac{1}{\alpha} \operatorname{Ln}\left[\frac{1+e^{\alpha(\Phi-\beta e V)}}{1+e^{\alpha[\Phi+(1-\beta) e V]}}\right]\right)
$$

where $\Phi$ is the barrier height (bottom of the first quantized level), $\alpha=t_{B} \pi^{2} h^{-1} \sqrt{2 m^{*} / \Phi}$ is a parameter related to the inverse of the potential barrier curvature (assuming a parabolic longitudinal potential), $m^{*}=0.44 m_{0}$ is the effective electron mass and $t_{B}$ is the barrier thickness at the equilibrium Fermi energy. $\beta$ takes into account how the potential drops at the two ends of the filament: $\beta=1$ has been used since the constriction is highly asymmetric [15]. $G / G_{0}$ is a conductance parameter equivalent to the number of filaments at very low voltages: in a very approximate way, a single highly conductive filament can be viewed as a parallel combination of elementary nanowires [17].

I-V Reset operation has different impacts from cell-to-cell, resulting either into a break or a modulation of the conductive filament $(\mathrm{CF})[8,15]$. In the former case the presence of a potential barrier is assumed, hence fitting is performed considering $G / G_{0}=1$ and the average barrier length $d$ and radius of the constriction $r$ are calculated according to [12]. In the latter case, assuming the absence of a potential barrier, the normalized conductance of the filament $G / G_{0}$ is calculated. The percentage of cells resulting either into a $\mathrm{CF}$ break or modulation are reported in Tab. 2: the high leakage current in P-array makes very difficult to completely interrupt the conductive path hence the lowest percentage of CF break is obtained, whereas 
Table 2: Reset condition comparison.

\begin{tabular}{|l|c|c|}
\hline Technology & C.F. Break [\%] & C.F. Modulation [\%] \\
\hline A, $1 \mu m^{2}$ & 45 & 55 \\
A, $0.4 \mu m^{2}$ & 34 & 66 \\
P $0.4 \mu m^{2}$ & 20 & 80 \\
\hline
\end{tabular}

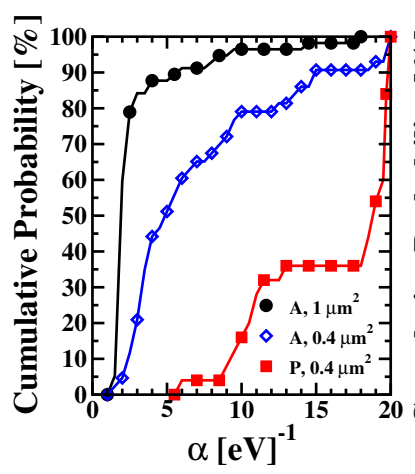

(a)

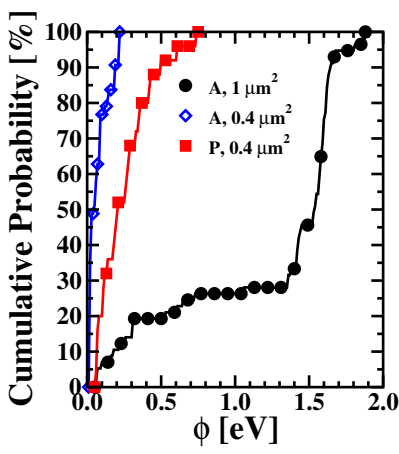

(b)
Figure 8: Cumulative distribution of $\alpha$ and $\Phi$ fitting parameters used on $\mathrm{CF}$ break cells.

Table 3: Average value and standard deviation of fitting parameters calculated on CF break cells.

\begin{tabular}{|l|c|c|c|c|}
\hline Technology & \multicolumn{2}{|c|}{$\alpha[\mathrm{eV}]^{-1}$} & \multicolumn{2}{c|}{$\phi[\mathrm{eV}]$} \\
\hline & avg. & std. & avg. & std. \\
\hline $\mathrm{A}, 1 \mu \mathrm{m}^{2}$ & 2.67 & 3.02 & 1.21 & 0.58 \\
$\mathrm{~A}, 0.4 \mu \mathrm{m}^{2}$ & 6.83 & 5.48 & 0.07 & 0.06 \\
$\mathrm{P}, 0.4 \mu \mathrm{m}^{2}$ & 16.08 & 5.06 & 0.17 & 0.25 \\
\hline
\end{tabular}

the highest percentage is obtained on A-array with the larger resistor area. The cumulative distributions of $\alpha$ and $\Phi$ fitting parameters calculated on the $\mathrm{CF}$ break cells are reported in Fig. 8.

Average value and standard deviation of the fitting parameters are reported in Tab. 3. The cumulative distributions of calculated barrier length $d$ and radius $r$ of the CF constriction are reported in Fig. 9, while the average value and standard deviations are reported in Tab. 4. A-array with the small resistor area shows the largest radius with the lowest barrier length: the presence of a very large constriction with a very low barrier explains the issues in controlling the cells' uniformity during Set and Reset operations. A-array with the larger resistor area shows higher barrier and smaller radius, resulting into a higher controllability during Set and Reset. Moreover, the highest parameters uniformity is observed, which translates into the highest HRS and LRS currents uniformity. P-array shows the largest barrier with the highest variability: the highest barrier is the reason of the higher average ratio between HRS and LRS, while the high variability generates the high current variability observed in HRS.

In case of CF modulation fitting has been performed assuming large negative $\Phi$ values, $\alpha$ fixed to 1 (even if $\alpha$ and $\Phi$ play no role in such condition) and $G / G_{0} \geq 1$ due to the presence of the residual filament. Fig. 10 shows the cumulative distribution of $G / G_{0}$ conductance values fitting parameters used on hard to

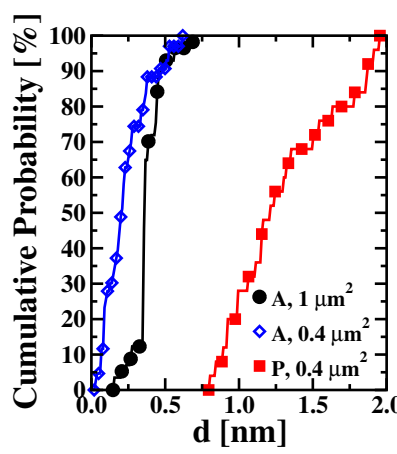

(a)

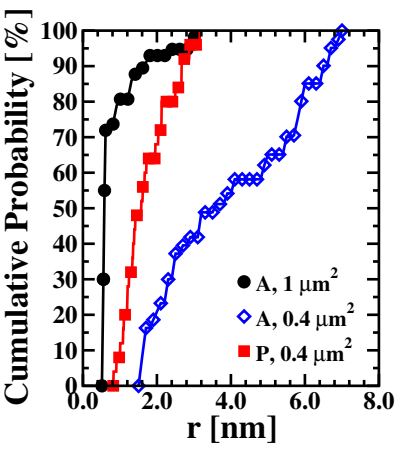

(b)
Figure 9: Cumulative distribution of calculated barrier length $d$ (a) and radius of the filament constriction $r$ (b) on CF break cells.

Table 4: Average value and standard deviation of barrier length and filament radius calculated on $\mathrm{CF}$ break cells.

\begin{tabular}{|l|c|c|c|c|}
\hline Technology & \multicolumn{2}{|c|}{$d[\mathrm{~nm}]$} & \multicolumn{2}{c|}{$r[\mathrm{~nm}]$} \\
\hline & avg. & std. & avg. & std. \\
\hline A, $1 \mu \mathrm{m}^{2}$ & 0.37 & 0.11 & 0.85 & 0.62 \\
$\mathrm{~A}, 0.4 \mu \mathrm{m}^{2}$ & 0.25 & 0.24 & 4.25 & 2.25 \\
$\mathrm{P}, 0.4 \mu \mathrm{m}^{2}$ & 1.29 & 0.36 & 1.71 & 0.65 \\
\hline
\end{tabular}

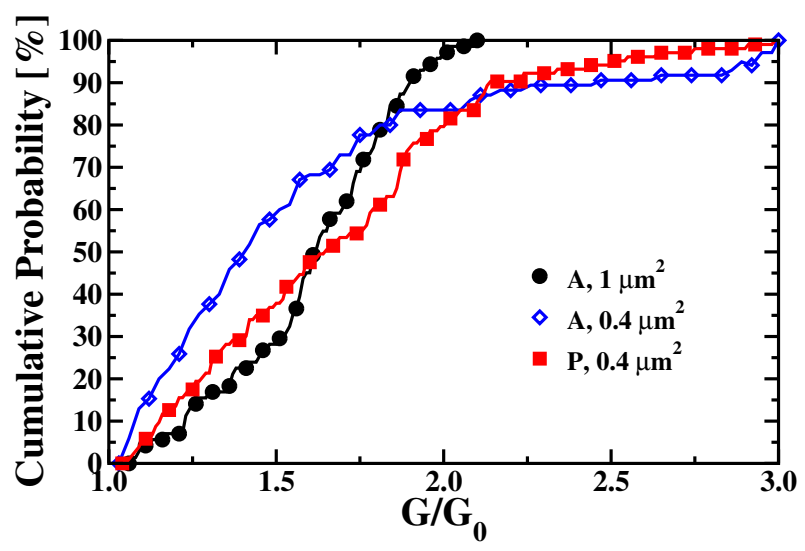

Figure 10: Cumulative distribution of $G / G_{0}$ fitting parameters used on hard to disrupt cells.

Table 5: $G / G_{0}$ average value and standard deviation.

\begin{tabular}{|l|c|c|}
\hline Technology & \multicolumn{2}{|c|}{$G / G_{0}$} \\
\hline & avg. & std. \\
\hline $\mathrm{A}, 1 \mu m^{2}$ & 1.61 & 0.26 \\
$\mathrm{~A}, 0.4 \mu m^{2}$ & 1.56 & 0.53 \\
$\mathrm{P}, 0.4 \mu m^{2}$ & 1.67 & 0.42 \\
\hline
\end{tabular}

disrupt cells: it can be observed that A-array with the larger resistor area shows the lowest variability, which is the reason of the lowest HRS current variability observed during Reset with the Incremental Pulse and Verify algorithm. Average value and standard deviation of the fitting parameter $G / G_{0}$ are reported in Tab. 5 . 


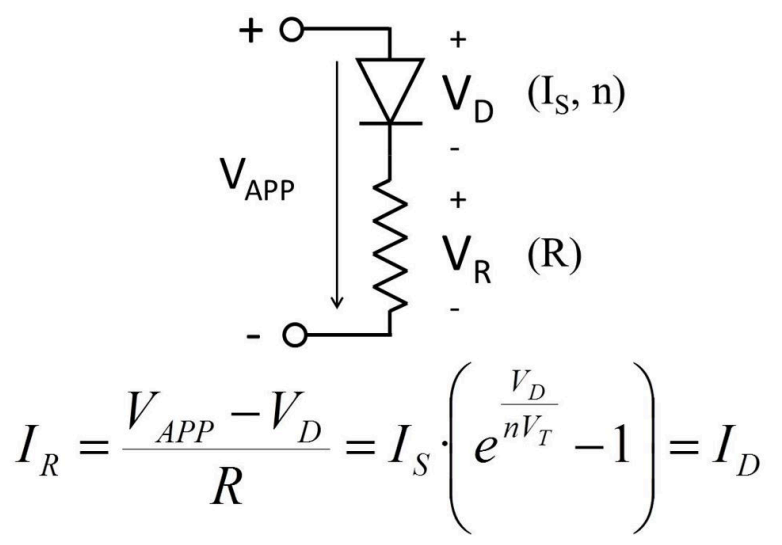

Figure 11: Equivalent model for the 1T-1R device based on a Diode-Resistor circuit. Resistance value $(\mathrm{R})$, saturation current $\left(\mathrm{I}_{s}\right)$ and ideality factor $(\mathrm{n})$ of the diode are the parameters used to fit the conduction of the 1T-1R devices at both resistive states, LRS and HRS, and for both types of samples, amorphous and poly-crystalline.

\subsection{Equivalent circuit modeling}

Electrical models are a power tool to analyze memory devices and circuits based on Resistive Switching (RS) devices allowing evaluating characteristics like power consumption or performance in large RS devices arrays $[16,18]$. To model the experimental I-V curves during both RS states (i.e., LRS and HRS) we use a Diode-Resistor based circuit Fig. 11 where the resistance value $(\mathrm{R})$, the diode saturation current $\left(\mathrm{I}_{s}\right)$ and diode ideality factor (n) are the parameters of the model [16]. $\mathrm{V}_{A P P}$ represents $\mathrm{V}_{B L}$ or $\mathrm{V}_{S L}$, which are the applied voltages to produce the Set and Reset processes respectively.

To fit the all the experimental curves an automatized process has been developed to extract the model parameters values for each curve. Fig. 12 shows some examples of experimental LRS curves (blue circles) before the reset process and the simulated curves using the circuit model of Fig. 11 with suitable parameters (red lines). As can be observed, the model fits perfectly with the experimental results for both amorphous and polycrystalline samples. For each kind of samples, the analyzed voltage range was limited by the reset voltage that is lower for the poly-crystalline sample. Larger current levels are obtained for the poly-crystalline sample.

The same automatic process was also used to fit HRS curves for both samples types. Fig. 13 shows experimental HRS curves (blue circles) before the set process and the corresponding simulated curves (red lines). Poly-crystalline samples show larger current levels than the amorphous ones which are very noisy at low voltages $(<1 \mathrm{~V})$. This noisy current observed in the amorphous samples, must be neglected to avoid errors during the fitting process. For this reason, current values for $V_{B L}$ below 1 $\mathrm{V}$ are not considered to force a better fitting for voltages larger than $1 \mathrm{~V}$, where the $\mathrm{I}-\mathrm{V}$ curve is not affected by the noise. This high noise level in the current at low voltages could be caused by the nature of the memory cell and the array structure where the drive transistor effect on the electrical characteristics of the memory must be analyzed in detail.

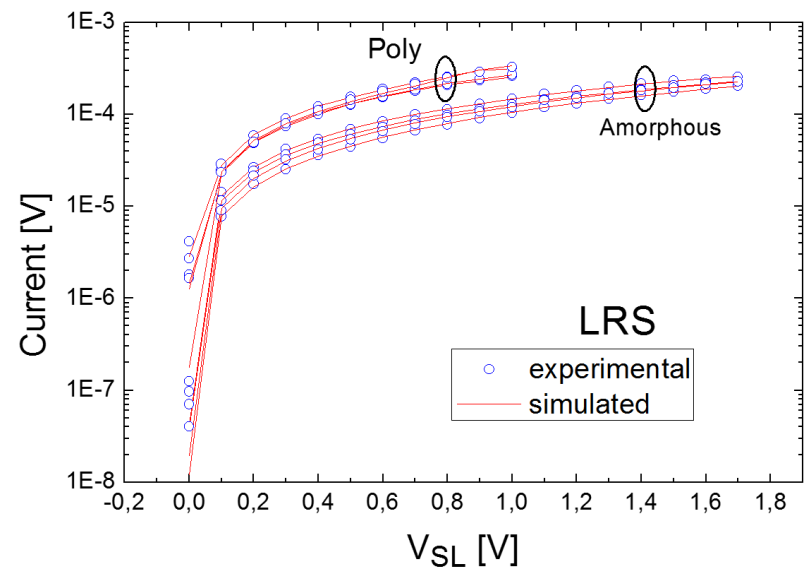

Figure 12: Experimental LRS I-V curves (blue circles) and the simulated curves (red lines) obtained using the Diode-Resistor model. With a suitable parameter set, the model reproduces properly the experimental curves for both polycrystalline and amorphous samples.

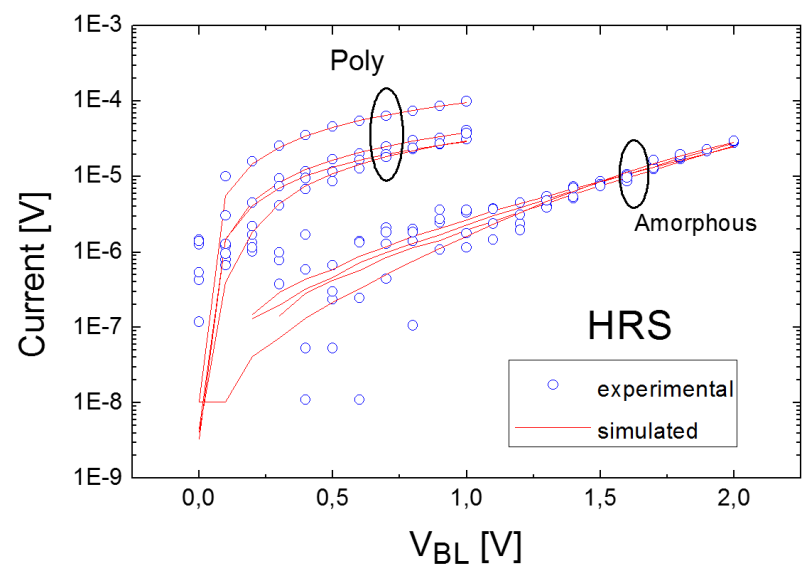

Figure 13: Experimental HRS I-V curves (blue circles) and simulated HRS curves (red lines) using the Diode-Resistor model for both amorphous and poly-crystalline samples. Noisy currents at low voltages cannot be fitted by the model, especially for the amorphous samples where current values for $\mathrm{V}_{B L}$ below $1 \mathrm{~V}$ are not considered.

\section{Conclusions}

1T-1R RRAM arrays manufactured with $\mathrm{P}-\mathrm{HfO}_{2}$ shows several advantages compared to $\mathrm{A}-\mathrm{HfO}_{2}$ even considering their improved process: higher current Ratio, lower switching voltages, lower power consumption, minor endurance degradation and higher overall yield. Moreover, P-array show very low $\mathrm{V}_{S E T}$ variability, hence faster Set operation could be reliably performed. P-array disadvantages are represented by the larger HRS distribution after Forming, the higher Reset voltage dispersion, the lower read disturb immunity and the higher $\mathrm{V}_{\text {FORM }}$ if compared to A-array with the same resistor area, however it must be pointed out that such operation is performed only once. The grain boundaries conduction mechanism in the polycrystalline $\mathrm{HfO}_{2}$ structure could be the reason of the higher cellto-cell variability observed in P-arrays. QPC modeling allowed showing that the higher uniformity observed on A-array with the large resistor area can be ascribed to a lower conductive filament shape variability in terms of radius of the constriction and 
barrier height, whereas the $\mathrm{P}$-array shows the highest variability in terms of conductive filament shape: the reason could be ascribed again to the different conduction mechanism and the higher leakage currents observed on such technology. A dioderesistor equivalent circuit model correctly fits the experimental RS I-V characteristics of poly-crystalline and amorphous samples for both LRS and HRS. However, noisy current levels at low voltages, especially for amorphous samples, could lead to a non-well fitted curve. Thus, it is needed to remove them for a suitable current fitting at larger voltages.

\section{Acknowledgments}

This work was supported by the European Union's H2020 research and innovation programme under grant agreement $\mathrm{N}^{o}$ 640073.

[1] S. Tanachutiwat, M. Liu, W. Wang, FPGA Based on Integration of CMOS and RRAM, IEEE Trans. on Very Large Scale Integration (VLSI) Systems 19 (11) (2011) 2023-2032. doi:10.1109/TVLSI.2010.2063444.

[2] D. Garbin, E. Vianello, O. Bichler, Q. Rafhay, C. Gamrat, G. Ghibaudo, B. DeSalvo, L. Perniola, HfO2-Based OxRAM Devices as Synapses for Convolutional Neural Networks, IEEE Trans. on Electron Devices 62 (8) (2015) 2494-2501. doi:10.1109/TED.2015.2440102.

[3] T. Xu, V. Leppanen, Analysing emerging memory technologies for big data and signal processing applications, in: Digital Information Processing and Communications (ICDIPC), 2015 Fifth International Conference on, 2015, pp. 104-109. doi:10.1109/ICDIPC.2015.7323014.

[4] C. Zambelli, A. Grossi, D. Walczyk, T. Bertaud, B. Tillack, T. Schroeder, V. Stikanov, P. Olivo, C. Walczyk, Statistical analysis of resistive switching characteristics in ReRAM test arrays, in: IEEE Int. Conf. on Microelectronics Test Structures (ICMTS), 2014, pp. 27-31.

[5] C. Zambelli, A. Grossi, P. Olivo, D. Walczyk, J. Dabrowski, B. Tillack, T. Schroeder, R. Kraemer, V. Stikanov, C. Walczyk, Electrical characterization of read window in ReRAM arrays under different SET/RESET cycling conditions, in: IEEE Int. Memory Workshop (IMW), 2014, pp. $1-4$.

[6] D. Walczyk, T. Bertaud, M. Sowinska, M. Lukosius, M. A. Schubert, A. Fox, D. Wolansky, A. Scheit, M. Fraschke, G. Schoof, C. Wolf, R. Kraemer, B. Tillack, R. Korolevych, V. Stikanov, C. Wenger, T. Schroeder, C. Walczyk, Resistive switching behavior in $\mathrm{TiN} / \mathrm{HfO}_{2} / \mathrm{Ti} / \mathrm{TiN}$ devices, in: Int. Semiconductor Conf. Dresden-Grenoble (ISCDG), 2012, pp. 143-146. doi:10.1109/ISCDG.2012.6360035.

[7] P. Lorenzi, R. Rao, F. Irrera, Forming kinetics in $\mathrm{HfO}_{2}$-based RRAM cells, IEEE Trans. on Electron Devices 60 (1) (2013) 438-443. doi:10.1109/TED.2012.2227324.

[8] A. Grossi, D. Walczyk, C. Zambelli, E. Miranda, P. Olivo, V. Stikanov, A. Feriani, J. Sune, G. Schoof, R. Kraemer, B. Tillack, A. Fox, T. Schroeder, C. Wenger, C. Walczyk, Impact of intercell and intracell variability on forming and switching parameters in rram arrays, IEEE Trans. on Electron Devices 62 (8) (2015) 2502-2509. doi:10.1109/TED.2015.2442412.

[9] K. Morgan, R. Huang, S. Pearce, C. de Groot, The effect of atomic layer deposition temperature on switching properties of HfOx resistive RAM devices, in: IEEE Int. Symp. on Circuits and Systems (ISCAS), 2014, pp. $432-435$.

[10] H.-D. Kim, F. Crupi, M. Lukosius, A. Trusch, C. Walczyk, C. Wenger, Resistive switching characteristics of integrated polycrystalline hafnium oxide based one transistor and one resistor devices fabricated by atomic vapor deposition methods, Journal of Vacuum Science and Technology B 33 (5) (2015) 052204.1-052204.5.

[11] A. Grossi, E. Perez, C. Zambelli, P. Olivo, C. Wengeru, Performance and reliability comparison of 1t-1r rram arrays with amorphous and polycrystalline $\mathrm{HfO}_{2}$, in: Joint Int. EUROSOI Workshop and Int. Conf. on Ultimate Integration on Silicon (EUROSOI-ULIS), 2016, pp. 80-83.

[12] E. A. Miranda, C. Walczyk, C. Wenger, T. Schroeder, Model for the resistive switching effect in $\mathrm{HfO}_{2}$ MIM structures based on the transmission properties of narrow constrictions, IEEE Electron Device Letters 31 (6) (2010) 609-611.

[13] A. Grossi, C. Zambelli, P. Olivo, E. Miranda, V. Stikanov, T. Schroeder, C. Walczyk, C. Wenger, Relationship among current fluctuations during forming, cell-to-cell variability and reliability in RRAM arrays, in: IEEE Int. Memory Workshop (IMW), 2015, pp. 1-4.

[14] V. Iglesias, M. Porti, M. Nafra, X. Aymerich, P. Dudek, G. Bersuker, Dielectric breakdown in polycrystalline hafnium oxide gate dielectrics investigated by conductive atomic force microscopy, Journal of Vacuum Science and Technology B 29 (1).

[15] A. Grossi, C. Zambelli, P. Olivo, E. Miranda, V. Stikanov, C. Walczyk, C. Wenger, Electrical characterization and modeling of pulse-based forming techniques in RRAM arrays, Solid-State Electronics 115, Part A (2016) 17 - 25. doi:http://dx.doi.org/10.1016/j.sse.2015.10.003.

[16] A. Crespo-Yepes, J. Martin-Martinez, I. Rama, M. Maestro, R. Rodriguez, M. Nafria, X. Aymerich., M. B. Gonzalez, F. Campabadal, Intradevice statistical parameters in variabilit-yaware modelling of resistive switching devices, in: Joint Int. EUROSOI Workshop and Int. Conf. on Ultimate Integration on Silicon (EUROSOI-ULIS), 2016, pp. 84-87.

[17] X. Lian, X. Cartoix, E. Miranda, L. Perniola, R. Rurali, S. Long, M. Liu, J. Su, Multi-scale quantum point contact model for filamentary conduction in resistive random access memories devices, Journal of Applied Physics 115 (24) (2014) 1-8.

[18] J. Martin-Martinez, B. Kaczer, R. Degraeve, P. J. Roussel, R. Rodriguez, M. Nafria, X. Aymerich, B. Dierickx, G. Groeseneken, Circuit design-oriented stochastic piecewise modeling of the postbreakdown gate current in MOSFETs: Application to ring oscillators, IEEE Trans. on Device and Materials Reliability 12 (1) (2012) 78-85. doi:10.1109/TDMR.2011.2162238. 